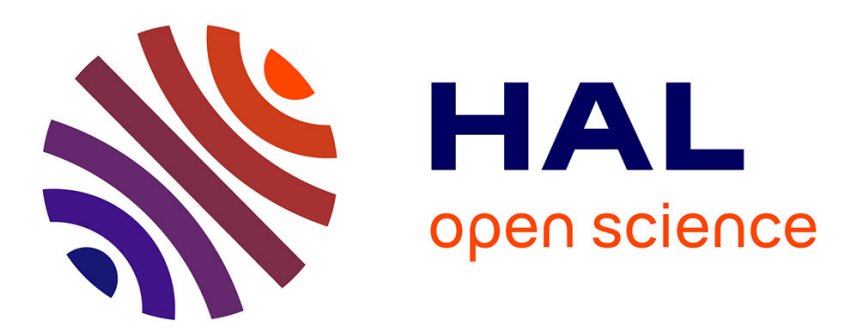

\title{
Optimization of Trailing-Edge Serrations to Reduce Open-Rotor Tonal Interaction Noise
}

\author{
Robert Jaron, Antoine Moreau, Sébastien Guérin, Rainer Schnell
}

\section{To cite this version:}

Robert Jaron, Antoine Moreau, Sébastien Guérin, Rainer Schnell. Optimization of Trailing-Edge Serrations to Reduce Open-Rotor Tonal Interaction Noise. 16th International Symposium on Transport Phenomena and Dynamics of Rotating Machinery, Apr 2016, Honolulu, United States. hal-01884252

\section{HAL Id: hal-01884252 \\ https://hal.science/hal-01884252}

Submitted on 30 Sep 2018

HAL is a multi-disciplinary open access archive for the deposit and dissemination of scientific research documents, whether they are published or not. The documents may come from teaching and research institutions in France or abroad, or from public or private research centers.
L'archive ouverte pluridisciplinaire HAL, est destinée au dépôt et à la diffusion de documents scientifiques de niveau recherche, publiés ou non, émanant des établissements d'enseignement et de recherche français ou étrangers, des laboratoires publics ou privés. 


\title{
Optimization of Trailing-Edge Serrations to Reduce Open-Rotor Tonal Interaction Noise
}

\author{
Robert Jaron ${ }^{1 *}$, Antoine Moreau ${ }^{1}$, Sébastien Guérin ${ }^{1}$, Rainer Schnell ${ }^{2}$
}

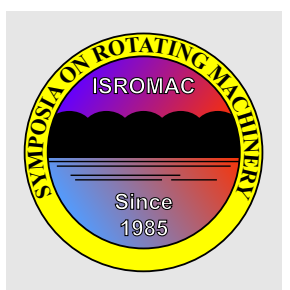

ISROMAC 2016

International

Symposium on Transport

Phenomena and

Dynamics of

Rotating Machinery

Hawaii, Honolulu

April 10-15, 2016

\begin{abstract}
A major source of contra-rotating open rotor tonal noise is caused by the interaction of the front-rotor wakes with the aft-rotor blades. Inspired by chevron nozzles, which increase the mixing process in jet shear layers, serrations are implemented at the front rotor trailing-edge in order to increase the wake mixing and thus reduce the tones. The depth and width of the serrations are optimized with a multiobjective, metamodel-assisted evolutionary algorithm. For each member a steady-state RANS (Reynolds Averaged Navier-Stokes) simulation is performed which is coupled with an analytical noise prediction method to evaluate the noise reduction due to the serrations. The results confirm that tonal interaction noise can be reduced by means of trailing-edge serrations. It is found that the major noise reduction mechanism for wake interaction is attributed to increased destructive interferences occurring in radial direction. The tonal noise, generated through the interaction of the rear rotor potential field with the front rotor trailing edge, is also slightly reduced because of the circumferential and axial shift of the serrated trailing edge. Furthermore the present study shows that an acoustical optimization can be successfully carried out with a hybrid approach that predicts the noise analytically and extracts the aerodynamic input data from a steady-state RANS flow solution.

\section{Keywords}

Trailing-Edge Serrations - Open Rotor - MDO - Tonal Interaction Noise

${ }^{1}$ Institute of Propulsion Technology, German Aerospace Center (DLR), Müller-Breslau-Str. 8, 10623 Berlin, Germany

${ }^{2}$ Institute of Propulsion Technology, German Aerospace Center (DLR), Linder Höhe, 51147 Cologne, Germany

*Corresponding author: robert.jaron@dlr.de
\end{abstract}

\section{INTRODUCTION}

Contra-rotating open rotors (CROR) have a high potential for fuel savings up to $25 \%$ compared to equivalent turbofan engines [1]. A drawback of the CROR configuration is the noise emission. Especially at take-off the requirement of low community noise combined with the need of high thrust is still a design challenge [2]. The rotor-rotor axial spacing is one of the key parameters that determines the emission of the wake interaction noise and potential field interaction noise [3]. However the rotor-rotor axial spacing is limited by integration and weight constraints with regard to the engine performance. Furthermore, the rear rotor can be cropped to prevent the interaction with the tip vortex shed from the front rotor [4]. In turn, the loading of the rear rotor have to be increased to preserve its performance which could result in an increase of the rear rotor self-noise and possibly of the wake interaction noise. As of today, studies are on going to further optimize rotor spacing and cropping by taking into account noise and performance constraints. The sweep of modern CROR blades also has several positive effects on the noise emission. With sweep the normal component of the relative Mach number is reduced so that no shock occurs at take-off. Moreover, swept blades can lead to reduced interaction noise due to the higher radial phase shift of the tilted wake interacting with the also swept rear rotor [5].

A further reduction of the tonal interaction noise is achievable by modifying the wakes shed from the front-rotor. With active systems, such as trailing-edge blowing tonal interaction noise can be reduced [6], but it is accompanied by a loss in aerodynamic efficiency.

Serrated trailing edges inspired by chevron nozzles are a passive method to reduce broadband self-noise. The noise reduction through chevron nozzles is achieved by reinforcing the mixing process and the turbulent energy transfer towards smaller eddies. Trailing edge serrations aim to reduce broadband self-noise by reducing the spanwise turbulence wall pressure correlation length. They are comprehensively investigated for single airfoils [7] [8], cascades [9] and rotating blades [10]. But regarding the reduction of tonal wake interaction noise through trailing-edge serrations there are barely any published studies.

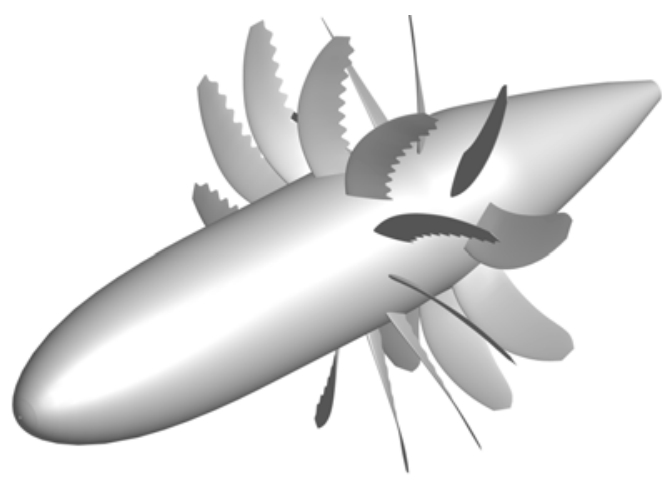

Figure 1. Contra-rotating open-rotor concept AI-PX7 designed by AIRBUS for JTI Clean Sky with serrations.

In 2004 Geiger [11] investigated fan blades in a linear cascade with serrated trailing-edges. Geiger found that the decay 
of the velocity deficit is faster in the presence of serrations. This effect is more pronounced with deeper serrations. The largest decay rate is found downstream of the serration tips while the lowest at the serration trough. Geiger concluded that the faster decay of the wakes will lead to a smaller unsteady loading of a blade downstream and therefore reduced tonal interaction noise.

In 2012 Weckmüller et al. [12] investigated the influence of trailing-edge serrations on tonal interaction noise of the contra-rotating open-rotor AI-PX7 designed by AIRBUS for JTI Clean Sky (see Fig. 1). The geometry is a generic design of a propulsion system for a 150 -seat, mid-range aircraft. Weckmüller introduced a process chain where the number and the depth of the serrations could be changed without changing the blade surface area in order to keep the aerodynamic performance constant. A constant aerodynamic performance, in particular a constant thrust with at least the same aerodynamic efficiency, is indispensable as any change of the thrust modifies the emitted sound power more than proportionally. One serrated configuration was simulated with a 3D unsteady RANS, and the acoustic far-field directivity was calculated using a variant of the Ffowcs-Williams and Hawkings acoustic analogy derived for the convective wave equation [13] [14]. A comparison with the reference geometry without serrations showed an overall power level (OAPWL) reduction of $0.2 \mathrm{~dB}$ for the tonal noise sources but also a slightly reduced thrust. Due to the high computational cost of an unsteady RANS simulation, no more configuration were investigated.

In the present study, the number and the depth of the trailing-edge serrations of the front rotor of the CROR AI-PX7 are optimized with a multi-disciplinary optimization process chain in order to reduce the tonal interaction noise. The acoustic cost function is calculated with an analytical noise prediction tool. The rotational speed is also optimized in order to keep the thrust and the aerodynamic efficiency at least at the same level of the reference configuration without serrations.

\section{PRESENTATION OF THE BASELINE CONFI- GURATION}

As baseline for the optimization the AI-PX7 full-scale geometry without serration at take-off conditions is used (hereafter referred to as "reference configuration"). The main characteristics of the AI-PX7 geometry and operating conditions are given in Tab. 1.

Table 1. AI-PX7 geometry and operating conditions

\begin{tabular}{llr}
\hline FR blade count $\left(B_{F R}\right)$ & {$[-]$} & 11 \\
RR blade count $\left(B_{R R}\right)$ & {$[-]$} & 9 \\
FR diameter $(D)$ & {$[\mathrm{m}]$} & 4.27 \\
RR cropping & {$[-]$} & $10 \%$ \\
\hline axial Mach number & {$[-]$} & 0.23 \\
relative tip Mach number & {$[-]$} & 0.75 \\
\hline
\end{tabular}

The aerodynamic performance parameters given in Tab. 2 are specified in terms of the nondimensional advance ratio $J$, the power coefficient $C_{P}$, the thrust coefficient $C_{T}$ and the efficiency $\eta$, as defined in Eq. (1) to (4):

$$
\begin{aligned}
& J=\frac{U_{\infty}}{n D} \\
& C_{P}=\frac{P_{\text {shaft }}}{\rho_{\infty} n^{3} D^{5}} \\
& C_{T}=\frac{T}{\rho_{\infty} n^{2} D^{4}} \\
& \eta=\frac{T U_{\infty}}{P_{\text {shaft }}}
\end{aligned}
$$

where $n$ gives the rotational speed in $1 / s, U_{\infty}$ stands for the velocity far upstream in $\mathrm{m} / \mathrm{s}, P_{\text {shaft }}$ denotes the shaft input power in $W$ and $T$ is the thrust in $N$.

Table 2. AI-PX7 performance at take-off

\begin{tabular}{lllll}
\hline & $J$ & $C_{P}$ & $C_{T}$ & $\eta$ \\
$\mathrm{FR}$ & 1.07 & 0.799 & 0.485 & 0.647 \\
$\mathrm{RR}$ & 1.19 & 1.012 & 0.550 & 0.643 \\
total & & & & 0.645 \\
\hline
\end{tabular}

Detailed information about the design and aerodynamics of the AI-PX7 CROR are given by Negulescu [15].

\section{OPTIMIZATION SETUP}

In the optimization loop the serrations are implemented according to the specifications given by the optimization algorithm. The mesh generation and the steady RANS simulation are performed automatically and each simulated member is acoustically evaluated with an analytical noise prediction tool (see Fig. 2). Moreau et al. [16] used the same approach to optimize the casing contour of a fan stage with respect to broadband interaction noise.

The aim of the optimization is to show a trend for the design of the serrations. Calculating an absolute noise reduction would require highly resolved unsteady simulations due to the high three dimensional behavior of the flow field behind the serrations.

\subsection{Optimization procedure}

The optimization is done with the DLR in-house multi-objective, metamodel-assisted evolutionary algorithm AutoOpti [17]. The metamodels are trained with all relevant values. New members are created through temporary optimizations of the metamodels, which are searching for a member with 


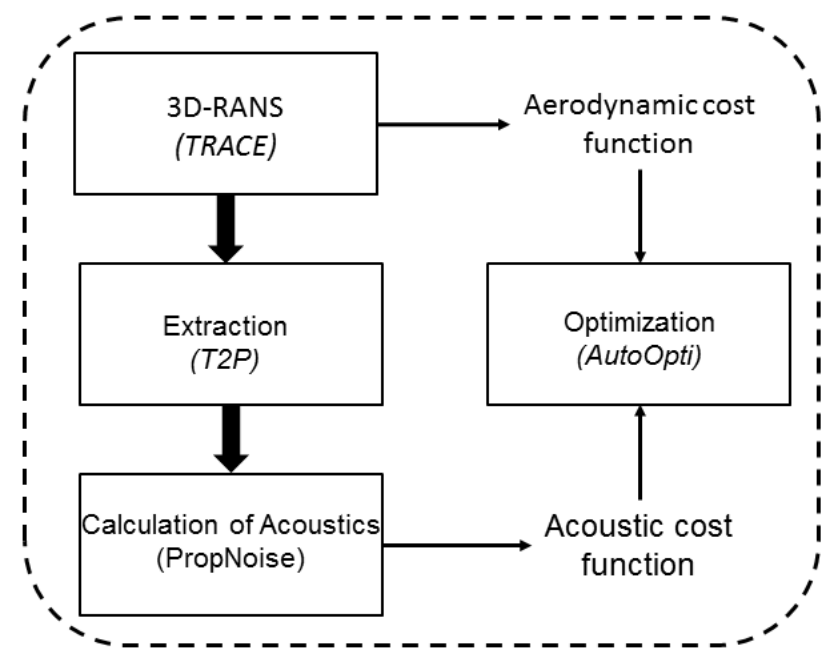

Figure 2. Process chain for the optimization.

the highest expected volume gain with respect to the actual Pareto front [18].

Although the changes of the serration shape are done at constant blade surface area, there are inevitably small variations in the thrust. The deficit of the thrust is mainly caused by total pressure losses due to the vortex shedding at the blunt trailing-edges at the trough of the serrations. The thickness of the blunt trailing-edge depends on the serration depth, and hence the thrust also varies with the serration depth. Lift noise changes approximately with the square or cube of the thrust, provided that the rotor diameter is constant. Therefore the thrust of the optimized configuration should be at least on the same level as for the reference configuration in order to be sure that the noise is reduced due to the serrations and not because of a lower thrust. Modern CROR engines operate at certain given rotational speeds and the thrust requirement is obtained by modifying the blade pitch angle which has an impact on the loading noise. As the used process chain does not enable the change of the pitch angle, the rotational speed is varied within the optimization loop until the constraint of the imposed thrust is met. A change of the rotational speed, even small, will have an impact on the self-noise sources. The parameter range of the optimization is specified in Tab. 3 .

Table 3. Table of optimization parameters

\begin{tabular}{ll}
\hline Parameter & Range \\
\hline serration number & $0-20$ \\
serration depth & $0-0.15$ chord length \\
rpm increase & $0-7.5 \%$ \\
\hline
\end{tabular}

\subsection{CFD simulation}

The numerical steady RANS simulations are performed with the DLR in-house CFD code TRACE [19] [20]. TRACE (Turbomachinery Research Aerodynamic Computational Enviroment) is a parallelized Navier-Stokes flow solver for structured and unstructured grids. The code solves the compressible Navier-Stokes equations in the relative frame of reference by using a multi-block approach. The governing equations are discretized in generalized coordinates about the cell centers by using the finite-volume method. Upwind-biased spatial differencing in conjunction with the flux-difference-splitting method of Roe [21] is used to evaluate the inviscid fluxes. Flux limiting is used to obtain smooth solutions in the vicinity of shocks, should they occur. Viscous terms are discretized by using second-order accurate central differences. The governing equations are integrated in time by using a direct (nonfactored) implicit dual-time stepping algorithm of secondorder accuracy. The coupling between adjacent blocks may be accomplished either in a one-to-one or in a patched manner. The turbulence is modeled with the two-equation Wilcox $k-\omega$ model. The mesh consists of 9.3 million cells and is presented in Fig. 3.

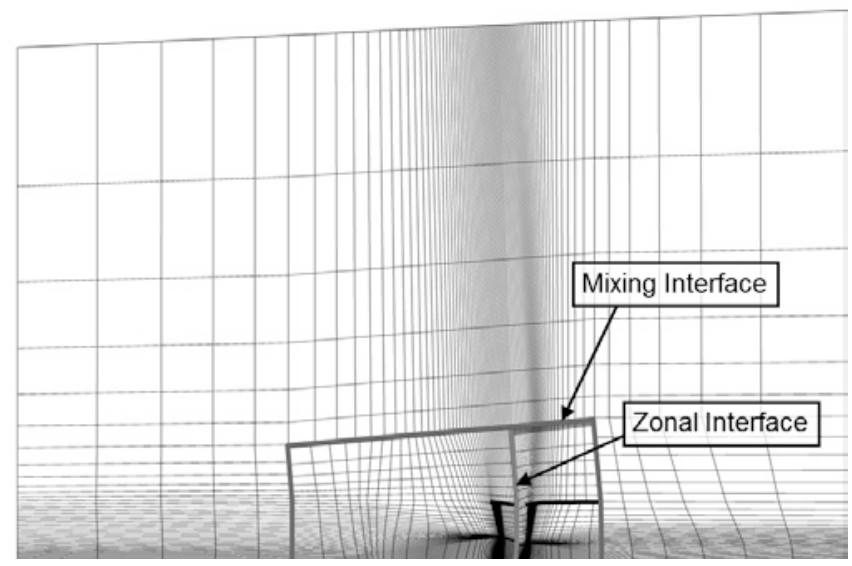

Figure 3. Mesh for the RANS simulation, one over four lines shown [12].

Thereof 5.6 million cells are used in the front rotor region in order to resolve the shape of the serrations and the shed wakes (see Fig. 4).

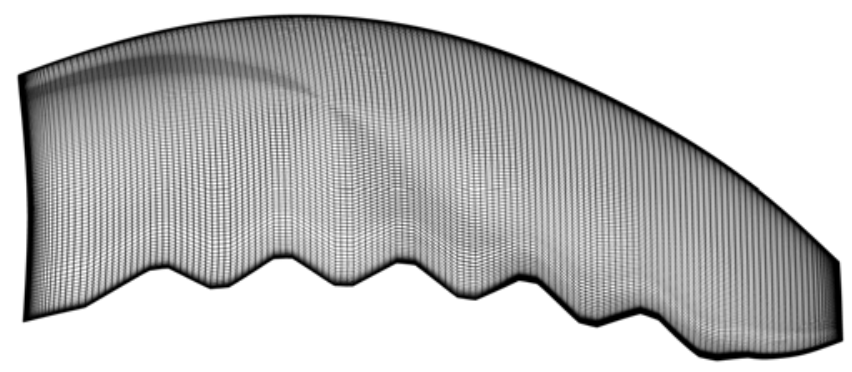

Figure 4. Blade surface mesh of the best member, every line shown.

The grid consists of three block groups: two rotating rotorblock groups and one non-rotating far-field block group. Only one passage per rotor is simulated with periodic conditions on the azimuthal boundaries and a mixing plane at the interface between the rotors. The simulation is performed with the 
full-scale geometry. The far-field temperature is set to ISA + $10 \mathrm{~K}$ and a homogeneous mean flow in direction of the engine axis with $\mathrm{M}=0.23$ is assumed in the entire domain, which represents a take-off at sea level conditions.

\subsection{Noise prediction}

The noise calculation within the optimization is done with the analytical in-house prediction tool PropNoise (acronym for Propulsion Noise). PropNoise predicts fan tonal and broadband noise and is based on the frequency formulation of the acoustic analogy applied to rotating blades. The analytical models of PropNoise are valid for open, ducted, single and contra-rotating configurations [22].

The circumferential Fourier coefficient of the acoustic pressure (also called modal pressure) for tonal noise is calculated within PropNoise as follows:

$$
p_{m}(\omega)=B \int g_{m} \cdot \mathfrak{R} \cdot \Psi \cdot \zeta \cdot e^{i k_{x} x+i m \Theta} \cdot d r
$$

where $B$ stands for the blade count, $g_{m}$ denotes the modal Green's function, $\mathfrak{R}$ denotes the acoustic radiation efficiency, $\Psi$ accounts for the non-compactness of the blade response and $\zeta$ represents the aerodynamic excitation pressure. The aerodynamic excitation pressure $\zeta$ is calculated as follows for the different tonal noise sources:

$$
\begin{array}{ll}
\text { thickness noise } & \zeta=\rho_{0} U_{0}^{2} \frac{\bar{t}}{c} \\
\text { steady lift } & \zeta=\frac{1}{2} \rho_{0} U_{0}^{2} C_{L} \\
\text { unsteady lift } & \zeta=\frac{1}{2} \rho_{0} U_{0}^{2} C_{L}(\tilde{\omega})
\end{array}
$$

The unsteady lift coefficient for the tonal interaction noise reads:

$$
C_{L}(\tilde{\omega})=2 \pi \frac{u_{n}(\tilde{\omega})}{U_{0}} S(\tilde{\omega})
$$

where $u_{n}$ is the velocity fluctuation normal to the blade chord, $U_{0}$ denotes the mean flow velocity at the leading edge and $S$ is the low-frequency approximation for the Sears function for incompressible flows [23]. The frequencies of the CROR tones are in a range where, according to Amiet [24], a highfrequency formulation should be used. The compressibility effects depend on the chord-based Helmholtz number $k c / 2$ and are therefore related to the compactness of the source. The non-compactness of the source is considered in the chordwise correlation function $\Psi$ that is dependent on the chord-based Helmholtz number (see Moreau and Guérin [22]). Jaron et al. [25] have shown that with the hybrid method of extracting aerodynamic excitations from a RANS simulation and calculating acoustics with Eq. 7 the OAPWL of the tonal sources is predicted for the AI-PX7 CROR with $0.5 \mathrm{~dB}$ difference compared to an unsteady RANS simulation with Ffowcs-Williams and Hawkings (FW-H) extrapolation. The comparison was done up to the engine order 49 as the mesh was suitable up to this frequency.

For the optimization, the aerodynamic input needed for the calculation of the blade response in the spanwise integral of Eq. 7, is extracted from the RANS simulation at 99 strips distributed equidistantly in the spanwise direction. The extraction of the wakes is done in front of the mixing plane as introduced by Guérin et al. [26]. An extrapolation of the wakes and the potential field beyond the mixing plane as proposed by Jaron et al. [27] was not suitable as the wakes induced by the serrated blades have a highly three-dimensional behavior, which violates the assumptions made for the wake extrapolation method. The extraction of the wakes in front of the mixing plane leads to an overestimation of the interaction noise source as shown in [25] but should be consistent for all members.

\section{RESULTS OF THE OPTIMIZATION}

The optimization was performed on the Linux cluster of the DLR Institute of Propulsion Technology. In total 124 members were generated. Only 78 of them met the constraint of the minimum thrust. Figure 5 shows a contour plot of the optimization area. In the optimization the engine orders up to 49 were considered for the tonal noise calculation for better comparability with the study done by Weckmüller et al. [12] In the optimization results a trend for the number and depth of the serrations can be identified. A serration number of 4 to 9 with large amplitude leads to reduced tonal noise emission. The calculated optimum is located at the border of the optimization area, which indicates that a higher tonal noise reduction could be achieved with larger serrations. An enlargement of the optimization area was not possible due to the chosen process chain for the generation of the serrations with a constant blade surface area. For the sake of completeness, it should be noted, that the results shown in Fig. 5 depend also on the thrust which is not shown in the plot. Although there was a constraint for the minimum thrust, a higher thrust than the one of the reference was allowed. This explains why there are different noise levels on the axis where the amplitude and/or the number of serrations are zero.

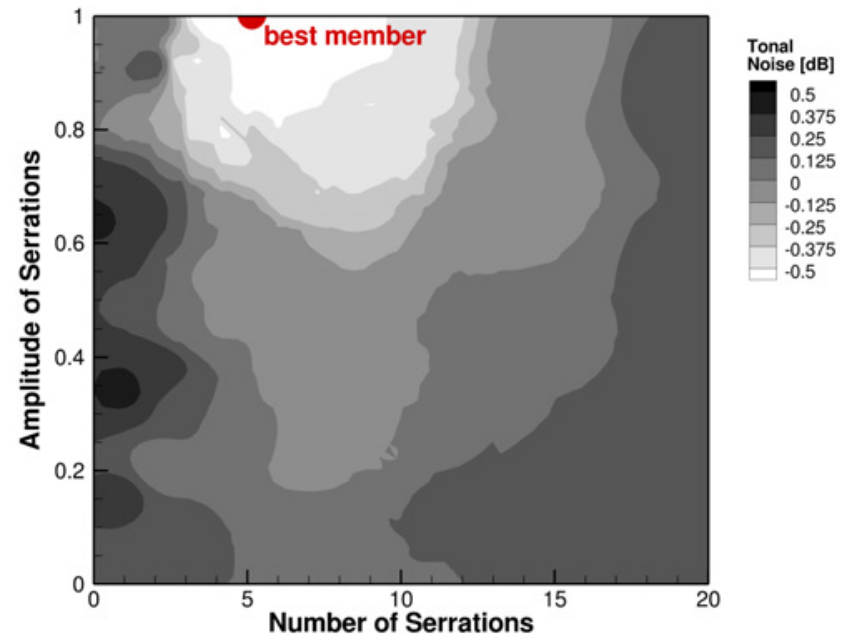

Figure 5. Tonal noise reduction in dependency of the serration number and depth.

A nondimensional paremeter based on the wavelength 
$\lambda$ of the tones and a characteristic length $l$ of the serrations (either the width or the depth) can be defined as follows:

$$
s=\frac{l}{\lambda}
$$

Considering the first interaction tone $(\mathrm{EO}=20)$ and the serration width, the nondimensional parameter $s_{w}$ varies in the optimization space from 0.99 to 0.05 . For the serration depth, the nondimensional parameter $s_{d}$ varies from 0 to 0.05 .

The member with the highest noise reduction highlighted as "best member" in Fig. 5 reached a reduction of $\sim 0.5 \mathrm{~dB}$ OAPWL (including tonal interaction noise and tonal selfnoise) with 5 serrations and a normalized amplitude of 1 , which equates to approximately 0.15 chord length. For this member the nondimensional parameters are $s_{w}=0.2$ and $s_{d}=0.05$ for $\mathrm{EO}=20$. By also considering higher engine orders up to 172, the noise reduction of the best member is even more pronounced - approximately $1 \mathrm{~dB}$ reduction with nearly the same total thrust and aerodynamic efficiency compared to the reference configuration (see Tab. 4). The aerodynamic performance and the acoustics of the best member (hereafter referred to as "serrated configuration") is given in detail in section 3.1 and section 3.2, respectively.

\subsection{Aerodynamic performance}

The rotational speed of both rotors was changed equally in the optimization in order to keep the rotational speed ratio and hence the angle of attack seen by the rear rotor constant. As the serrations lead to a thrust reduction of the front rotor and the total thrust was kept constant in the optimization, a slight shift of the thrust from the front rotor to the rear rotor is found for the best member with serrations as can be seen in Tab. 4. A reduced thrust of the front rotor could also be a reason for the reduced tonal interaction noise due to weaker wakes. In section 3.3 it will be shown that the reduced thrust of the front rotor is not the dominant noise cancellation mechanism.

Table 4. Performance of the serrated configuration relative to the reference configuration

\begin{tabular}{lllll}
\hline & $C_{P}$ & $C_{T}$ & $\eta$ & Thrust \\
FR & $-2.88 \%$ & $-2.06 \%$ & $+0.15 \%$ & $-0.86 \%$ \\
RR & $-1.18 \%$ & $-0.55 \%$ & $0.0 \%$ & $+0.72 \%$ \\
total & & & $+0.15 \%$ & $-0.18 \%$ \\
\hline
\end{tabular}

The modification of the radial distribution of the thrust through the serrations and the increased rotational speed is shown in Fig. 6. The impact of the serrations on the thrust is more pronounced in the outer part of the blade.

\subsection{Tonal noise}

In the optimization tonal interaction noise sources as well as tonal self-noise sources are considered. Fig. 7 shows the impact of the serrations on the different sources. The wake interaction noise is reduced through the serrations. The selfnoise sources are slightly higher for the serrated configuration

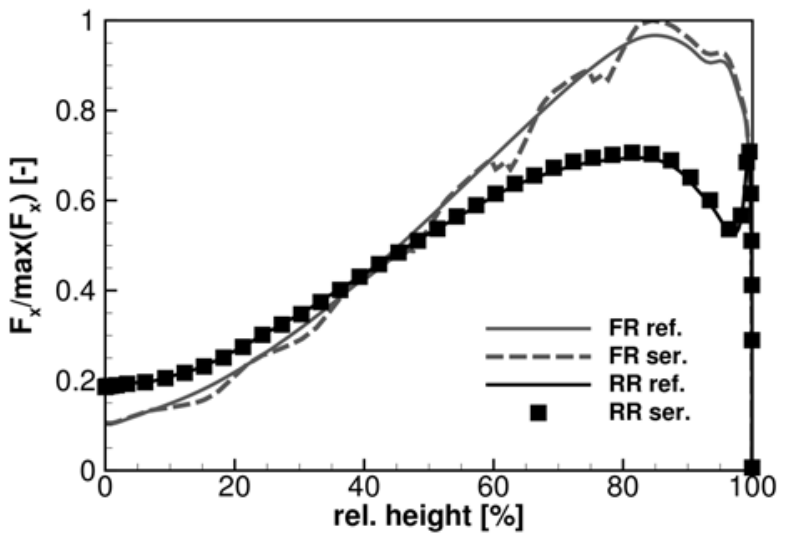

Figure 6. Modification of the radial distribution of the thrust through the serrations.

due to the slightly increased rotational speed. An additional benefit of the serrations is the reduction of the interaction noise generated by the potential field of the rear rotor interacting with the serrated trailing edge of the front rotor. The reduction is caused by the spanwise phase and axial shift of the trailing edge through the serrations which is accounted for in Eq. 7 with the exponential term $e^{i k_{x} x_{T E}+i m \Theta_{T E}}$. As the wake interaction source is clearly the dominant tonal noise source only this noise source will be further investigated.

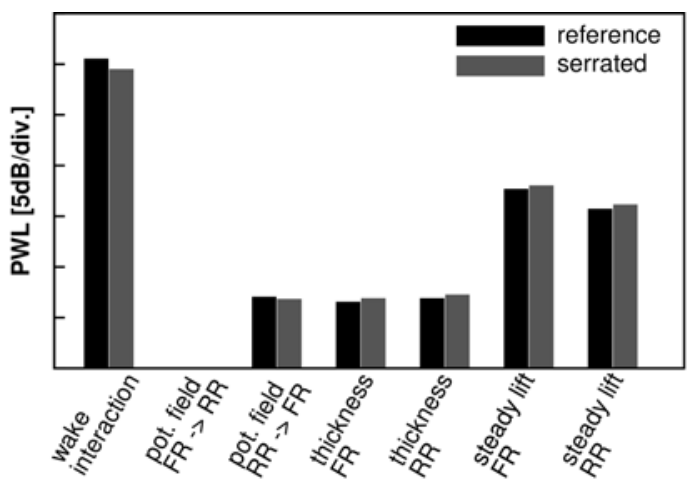

Figure 7. Impact of the serrations on the different tonal noise sources.

Figure 8 shows the interaction tones radiated from the reference configuration and from the optimized serrated configuration up to an engine order of 82 . For this configuration only one azimuthal mode is excited for each tone. Engine order 20 (BPF1+BPF2) and 29 (BPF1+2xBPF2) are dominant and have a higher sound power level with serrations than without. For most of the higher frequencies the sound power level is reduced with serrations.

For the engine orders 20 to 82 shown in Fig. 8 the ratio between the serration width $s_{w}$ and the wavelength varies from 0.2 to 0.8 and for the serration depth $s_{d}$ from 0.05 to 0.2 . With higher frequencies the acoustic wavelengths become smaller and therefore the ratio between the characteristic length of the serrations and the wavelengths gets larger and thus the 


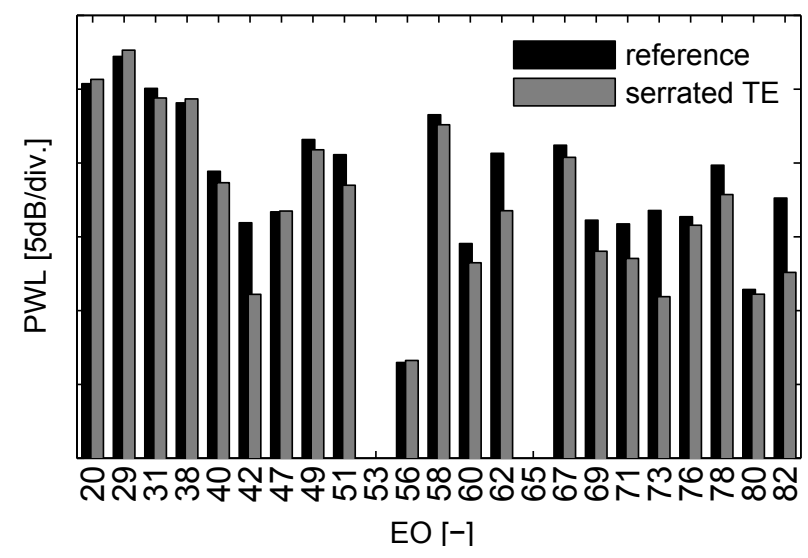

Figure 8. Tonal interaction noise of the reference and the serrated configuration

noise cancellation gets stronger. The analytically calculated spectra and the trend of increasing PWL reduction with higher frequencies agree with the results that Weckmüller et al. [12] found with unsteady RANS and FW-H.

\subsection{Noise Cancellation Mechanism}

The primary idea for introducing serrations was to accelerate the mixing of the wakes and to thereby achieve a reduction of the wake interaction noise. The wake shapes of the reference and serrated configurations shown in Fig. 9 indicate that there is also a variation of the wake phase along the span due to the change of the flow turning as a result of the serrations. The radial variation of the wake phase can lead to radial interferences which can cause a reduction of the emitted sound power.
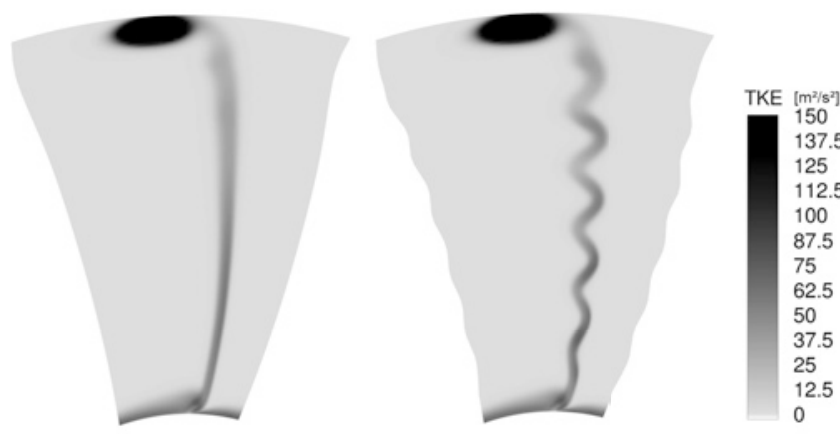

Figure 9. Shape of the wake of the reference (left) and serrated configuration (right) slightly upstream of the mixing plane.

The noise cancellation mechanism for the tonal interaction noise is investigated by comparing the magnitude and the phase angle of the unsteady lift of the serrated and the reference configuration at the example of EO $42(3 \times B P F 1+1 \times B P F 2)$ in Fig. 10. At this frequency the reduction is very pronounced (see Fig 8) and it can therefore be used as a good example for identifying the dominant noise cancellation mechanism. The amplitude of the unsteady lift of the serrated configuration oscillates around the unsteady lift of the configuration without serrations. Also the phase angle of the unsteady lift of the serrated configuration oscillates considerably more than the one of the reference.
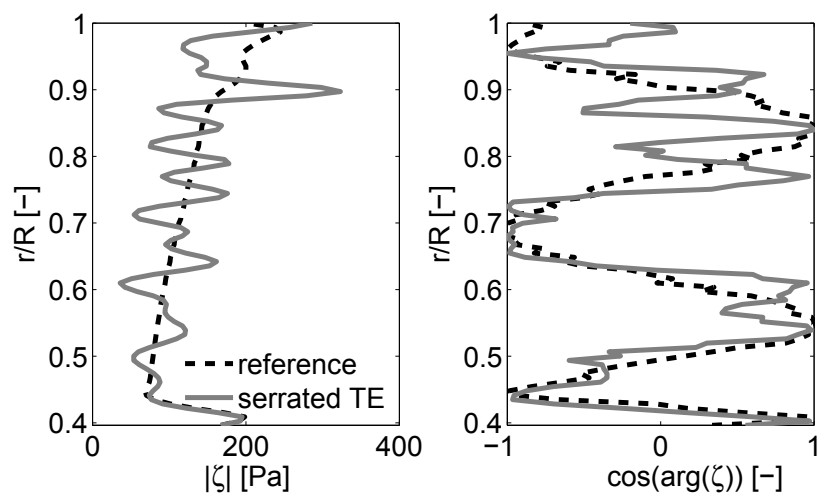

Figure 10. Comparison of the amplitude (left) and the phase angle (right) of the unsteady lift with and without serrations.

The impact of the changed magnitude and phase angle of the unsteady lift on the radiated noise can be investigated by neglecting all parts of Eq. (7) which are not influenced by the serrations. It only remains the complex unsteady lift integrated over the span (the integral is replaced by a discrete sum):

$$
I_{\zeta}=\frac{1}{N}\left|\sum_{k} \zeta_{k}\right|^{2}
$$

The integral is squared as the sound power level is proportional to the square of the modal pressure. Converting this integral into decibels a difference of $2.6 \mathrm{~dB}$ between the reference and the serrated configuration is achieved. The obtained PWL reduction for EO 42 shown in Fig. 8 is 4.8 dB. As the other terms of Eq. (7) are not influenced by the serrations, they just enhance the change of the unsteady lift.

To investigate the impact of the changed amplitude of the unsteady lift, the squared magnitude of the unsteady lift over the radius is integrated (again, the integral is replaces by a discrete sum):

$$
I_{|\zeta|}=\frac{1}{N} \sum_{k}\left|\zeta_{k}\right|^{2}
$$

Converting this integral into decibels a difference of only 0.05 $\mathrm{dB}$ is achieved. This clearly indicates that the noise reduction cannot be essentially attributed to a reduction in amplitude of the unsteady lift. In other words, the wake mixing is not considerably enhanced through the serrations.

In [28] a method was introduced to identify the impact of the radial interferences by considering the outer-product matrix of the radial complex distribution of the unsteady lift $\zeta$. By building this matrix it is possible to relate the integral $I_{\zeta}$ of Eq. (8) to the integral $I_{|\zeta|}$ of Eq. (9): 


$$
\begin{aligned}
& \frac{1}{N}\left|\sum_{k} \zeta_{k}\right|^{2}=\left(\frac{1}{N} \sum_{k}\left|\zeta_{k}\right|^{2}\right) \cdot\left(\frac{1}{N} \sum_{i, j} X_{i j}\right) \\
& \text { where } \quad X_{i j}=\frac{\operatorname{Re}\left(\zeta_{i} \zeta_{j}^{*}\right)}{\frac{1}{N} \sum_{k}\left|\zeta_{k}\right|^{2}}
\end{aligned}
$$

As explained in [28] the non-dimensional coefficients $X_{i j}$ are elements of the outer-product matrix that is denoted as "radial interference matrix" hereafter. The case $X_{i j}>0$ means that the two radial positions with index $i$ and $j$ generate a constructive interference with respect to acoustics. On the contrary $X_{i j}<0$ corresponds to a destructive interference that reduces the magnitude of the integral $I_{\zeta}$ and eventually the emitted sound power. The normalization of the cross-spectrum matrix is such that $\left|X_{i j}\right| \sim 1$ for substantial interferences and $\left|X_{i j}\right|<<1$ for negligible terms.

Figure 11 shows the radial interference matrices of the unsteady lift of EO 42 for the reference and serrated configuration. Constructive interferences are colored in red and destructive interferences are colored in blue. For the reference configuration, there are mostly constructive interferences. For the serrated configuration, there is a periodic pattern of constructive and destructive areas. On the right-hand side of Fig. 11, the difference of the radial interference matrices of the reference and serrated configuration is shown. Blue indicates areas where the serrated configuration has more destructive interferences than the reference configuration and red areas indicates that the serrated configuration has more constructive interferences. For EO 42 the serrations clearly lead to more destructive interferences in the tip region. Since the amplitude of the unsteady lift gets higher from hub to tip (see Fig. 10), the destructive interferences in the tip region have a strong impact on the emitted sound power.
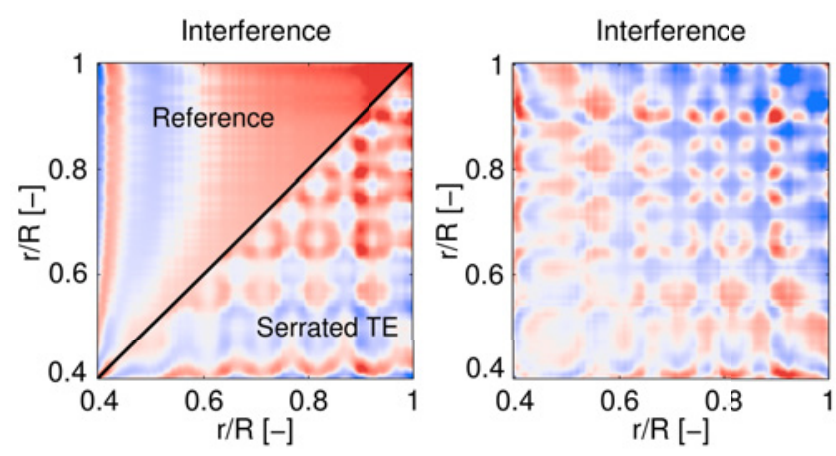

Figure 11. Radial interference matrices of the unsteady lift for the reference and serrated configuration (left) and the difference of both (right) at EO 42.

By converting the discrete sum of the radial interference matrix $X_{i j}$ into decibels, a reduction through the serrations of $2.55 \mathrm{~dB}$ is achieved due to radial destructive interferences. This matches very well with the sound power reduction of 2.6 $\mathrm{dB}$ mentioned above.

In Fig. 12 the difference of the tonal interaction noise between the reference and the serrated configuration is split into

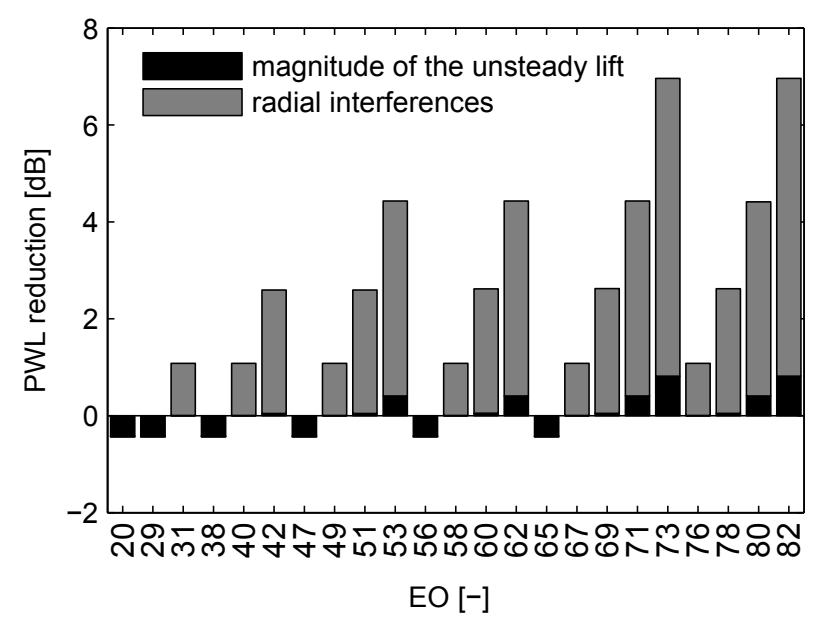

Figure 12. Respective contributions of unsteady-lift magnitude reduction (black) and radial interferences (gray) to the sound power reduction.

the parts that result from the change of the magnitude of the unsteady lift and from radial interferences. The radial interferences are clearly the dominant noise cancellation mechanism. For the lower frequencies the magnitude of the unsteady lift is amplified which leads to an increase of the sound power level. The increased unsteady-lift amplitude indicates that the wakes are enforced due to the blunt trailing-edges at the serrations troughs as shown in Fig. 13.

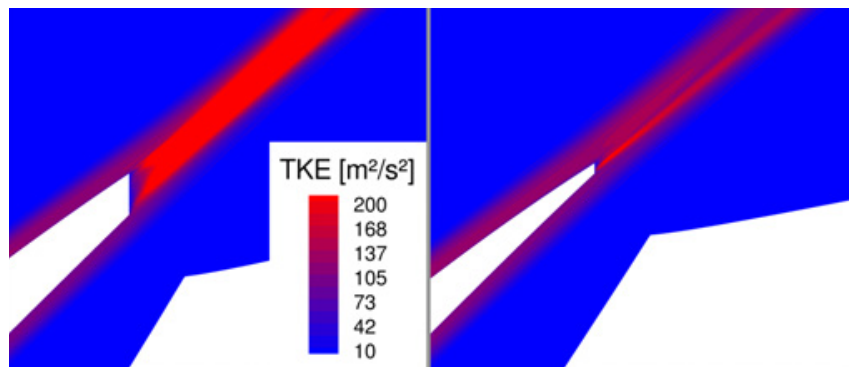

Figure 13. Wake at the blunt trailing-edge at the serration trough (left) and at the serration tip (right)

The results shown in Fig. 12 are consistent with the sound power spectrum shown in Fig. 8: the tones at lower frequencies are amplified and some of the higher frequency tones are strongly weakened due to radial interferences. That proves that it is sufficient for this case to investigate only the unsteady lift $\zeta$ and to neglect the other terms of Eq. (7) in order to understand the noise cancellation mechanism.

\section{SUMMARY AND CONCLUSION}

An automatic optimization of the number and depth of trailingedge serrations for the front-rotor of a contra-rotating open rotor configuration has been carried out in order to reduce the tonal noise. The noise calculation was done with a hybrid approach, which predicts the noise analytically and extracts the 
aerodynamic input data from a steady-state RANS flow solution. The present study has shown that tonal interaction noise can be reduced with trailing-edge serrations at take-off condition by roughly $1 \mathrm{~dB}$ without deteriorating the aerodynamic performance. A trend for the number and the depth of the serrations has been identified. It has been shown that the primary noise cancellation mechanism is attributed to increased destructive interferences occurring in spanwise direction due to the spanwise variation of the wake phase angle. The spanwise phase angle variations are a result of the changed flow turning through the serrations.

Furthermore the present study has shown the potential of using analytical prediction methods in an acoustical optimization to identify noise reducing solution in the early design of turbomachinery.

\section{OUTLOOK}

The present study was a pilot application in two manners: the first application of the RANS-informed analytical noise prediction within an optimization process chain and the barely known impact of the serrations on the interaction tones. There are several possible improvements for future studies on trailingedge serrations:

1. According to Parry et al. [29] broadband noise is an important noise source for modern CROR designs. As the broadband noise is also influenced by the serrations, a better evaluation of the overall noise emission would be possible by also considering the broadband noise in the optimization.

2. The procedure of cutting the serrations into the blade used in the presented study leads to thick blunt trailingedges at the serrations trough which generates larger velocity deficits and vortex shedding (see Fig. 13). The unsteadiness of the vortices can not be reproduced with steady simulations and is potentially underestimated in this study. To reduce the losses caused by the thick blunt trailing-edges, a better approach should be used to implement the serrations with thinner trailing-edges.

3. The depth and the width of the serrations was chosen to be constant about the blade height in this study. By introducing a variable depth and width over the span, the radial destructive interferences could be enhanced.

4. Probably more noise reduction could be achieved by increasing the parameter range concerning the serration depth as the highest noise reduction was achieved at the highest allowed serration depth.

5. It would be desirable to find an optimal value for the ratio of the acoustic wavelength and the characteristic length of the serration that would indicate how the serrations should be designed if a certain acoustic frequency is targeted to be reduced. Unfortunately the parameter space was too small in this study. Therefore it was not possible to derive such a similarity parameter based on this data set.

6. The used blade response function does not account for airfoil sweep and chord variations in the span-wise direction. This may have an impact on the noise calculation, particularly for noise sources located on the serrated front rotor. A more precise analytical calculation of the unsteady lift on the blade surfaces could be achieved by using a more complex formulation of the blade response function as for example proposed by Carazo et al. [5].

7. A further improvement of the blade response function could be achieved by using an improved model for the calculation of the interaction of the tip vortex with the rear rotor as proposed by Quaglia et al. [30].

8. The introduction of serrations at the leading edge of the rear rotor could also produce radial interferences. Furthermore, a combination of serrations on both, front and rear rotor, could enhance the radial interferences and therefore enhance the reduction of the tonal interaction noise.

\section{ACKNOWLEDGMENTS}

The authors would like to acknowledge the contribution of their colleague Henry Knobbe who helped to set up the optimization process chain and AIRBUS for the fruitful collaboration on the open-rotor topic engaged in the framework of Clean Sky Joint Technology Initiative (FP7) supported by the European Union.

\section{REFERENCES}

[1] S. Guérin, R. Schnell, and R. G. Becker. Performance prediction and progress towards multi-disciplinary design of contra-rotating open rotors. The Aeronautical Journal, 118(1208):1159-1179, October 2014.

[2] C. Hall, A. Zachariadis, T. Brandvik, and N. Sohoni. How to improve open rotor aerodynamics at cruise and takeoff. The Aeronautical Journal, 118(1208):1103-1123, October 2014.

[3] J. H. Dittmar, B. G. Eliott, and R. J. Jeracki. The Effect of Front-to-Rear Propeller Spacing on the Interaction Noise at Cruise Conditions of a Model Counterrotation Propeller having a Reduced Diameter Aft Propeller. Number NASA TM-101329 in Technical Report, October 1988.

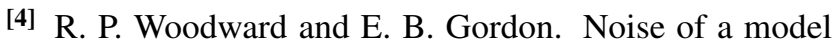
counterrotation propeller with reduced aft rotor diameter at simulated takeoff/approach conditions (F7/A3). Number AIAA-88-0263 in AIAA 26th Aerospace Sciences Meeting, Reno, Nevada, 11 - 14 January 1988.

[5] A. Carazo, M. Roger, and M. Omais. Analytical Prediction of Wake-Interaction Noise in Counter-Rotation Open Rotors. Number AIAA 2011-2758 in 17th AIAA/CEAS Aeroacoustics Conference, Portland, Oregon, June 2011.

[6] R. A. Akkermans, A. W. Stuermer, and J. Delfs. Assessment of Front-Rotor Trailing-Edge-Blowing for the Reduction of Open Rotor Noise Emissions. Number AIAA 2013-2200 in 19th AIAA/CEAS Aeroacoustics Conference, Berlin Germany, June 2013. 
[7] M. S. Howe. Aerodynamic noise of a serrated trailing edge. Journal of Fluids and Structures, 5(1):33-45, 1991.

[8] M. Gruber, P. Joseph, and T. Chong. Experimental Investigation of Airfoil Self Noise and Turbulent Wake Reduction by the use of Trailing Edge Serrations. Number AIAA 2010-3803 in 16th AIAA/CEAS Aeroacoustics Conference, Stockholm, Sweden, 2012.

[9] A. Finez, M. Jacob, M. Roger, and E. Jondeau. Broadband Noise Reduction Of Linear Cascades With Trailing Edge Serrations. Number AIAA 2011-2874 in 17th AIAA/CEAS Aeroacoustics Conference 32nd, Portland, Oregon, June 2011.

[10] S. Sinayoko, M. Azarpeyvand, and B. Lyu. Trailing edge noise prediction for rotating serrated blades. 20th AIAA/CEAS Aeroacoustics Conference, Atlanta, GA, June 2014.

[11] D. H. Geiger. Comparative Analysis of Serrated Trailing Edge Designs on Idealized Aircraft Engine Fan Blades for Noise Reduction. Master's Thesis, 2004.

[12] C. Weckmüller and S. Guérin. On the influence of trailing-edge serrations on open-rotor tonal noise. 18th AIAA/CEAS Aeroacoustics Conference (33rd AIAA Aeroacoustics Conference), Colorado Springs, CO, June 2012.

[13] C. Weckmüller, J. Wellner, S. Guérin, and U. Michel. Ffowcs Williams \& Hawkings Formulation for the Convective Wave Equation and Permeable Data Surface. Number AIAA 2010-3710 in 16th AIAA/CEAS Aeroacoustics Conference, Stockholm, Sweden, June 2010.

[14] C. Weckmüller and S. Guérin. Highly-Efficient Hybrid CFD/FW-H Approach for Open-Rotor Tonal Computation. 14th CEAS-ASC Workshop \& 5th Scientific Workshop of X3-Noise, Aeroacoustics of high-speed aircraft propellers and open rotors, Warsaw, Poland, 7. October 2010.

[15] C. A. Negulescu. Airbus AI-PX7 CROR Design Features and Aerodynamics. SAE International Journal of Aerospace, 6(2):626-642, 2013.

[16] A. Moreau, A. L. Aulich, R. Jaron, E. Nicke, L. Enghardt, and $\mathrm{H}$. Knobbe-Eschen. Optimization of casing contours in an aero-engine fan stage with emphasis on rotor-stator interaction noise. ISROMAC-2016, International Symposium on Transport Phenomena and Dynamics of Rotating Machinery, Honolulu, Hawaii, USA, April 2016.

[17] C. Voß, M. Aulich, B. Kaplan, and E. Nicke. Automated Multiobjective Optimisation in Axial Compressor Blade Design. Number GT2006-90420 in ASME Turbo Expo, Barcelona, Spain, May 2006.

[18] C. Voß, M. Aulich, and T. Raitor. Metamodel Assisted Aeromechanical Optimization of a Transonic Centrifugal Compressor. 15th International Symposium on Transport Phenomena and Dynamics of Rotating Machinery, Honolulu, Hawaii, USA, February 2014.
[19] D. Nürnberger, F. Eulitz, S. Schmitt, and A. Zachcial. Recent progress in the Numerical Simulation of unsteady viscous multistage turbomachinery flow. ISABE 20011081, Bangalore, September 2001.

[20] H. Yang, D. Nürnberger, and H. P. Kersken. Toward Excellence in Turbomachinery Computational Fluid Dynamics: A Hybrid Structured-Unstructured ReynoldsAveraged Navier-Stokes Solver. Journal of Turbomachinery, 128(2):390, 2006.

[21] P. L. Roe. Approximate Riemann solvers, parameter vectors, and difference schemes. Journal of Computational Physics, 43(2):357-372, 1981.

[22] A. Moreau and S. Guérin. Similarities of the free-field and in-duct formulations in rotor noise problems. Number AIAA 2011-2759 in 17th AIAA/CEAS Aeroacoustics Conference, Oregon Portland, June 2011.

[23] W. R. Sears. Some Aspects of Non-Stationary Airfoil Theory and Its Practical Application. Journal of the Aeronautical Sciences, 8(3):104-108, 1941.

[24] R.K. Amiet. High frequency thin-airfoil theory for subsonic flow. AIAA Journal, 14(8):1076-1082, 1976.

[25] R. Jaron, A. Moreau, and S. Guérin. Extrapolation of RANS flow data for improved analytical fan tone prediction. 21th AIAA/CEAS Aeroacoustics Conference, Dallas Texas (USA), June 2015.

[26] S. Guérin, A. Moreau, C. Menzel, and C. Weckmüller. Open-rotor noise prediction with a RANS-informed analytical method. Number AIAA 2012-2303 in Proceedings of the 18th AIAA/CEAS Aeroacoustics Conference, Colorado Springs, Colorado, USA, June 2012.

[27] R. Jaron, A. Moreau, and S. Guérin. RANS-informed fan noise prediction: separation and propagation of rotor wake and potential field. 20th AIAA/CEAS Aeroacoustics Conference, Atlanta, June 2014.

[28] L. Enghardt, P. Kausche, A. Moreau, and T. Carolus. Active Control of Fan Tones by means of Trailing Edge Blowing. Number AIAA 2015-2828 in 21st AIAA/CEAS Aeroacoustics Conference, Dallas Texas (USA), June 2015.

[29] A. Parry, M. Kingan, V. Blandeau, and B. Tester. Relative importance of open rotor tone and broadband noise sources. Number AIAA 2011-2763 in 17th AIAA/CEAS Aeroacoustics Conference (32nd AIAA Aeroacoustics Conference), Portland, Oregon, June 2011.

[30] M. Quaglia, S. Moreau, M. Roger, and R. Fernando. A 3D analytical approach for Open rotor Blade Vortex Interaction(BVI) tonal noise. Number AIAA 2015-2984 in 21st AIAA/CEAS Aeroacoustics Conference, Dallas Texas (USA), June 2015. 